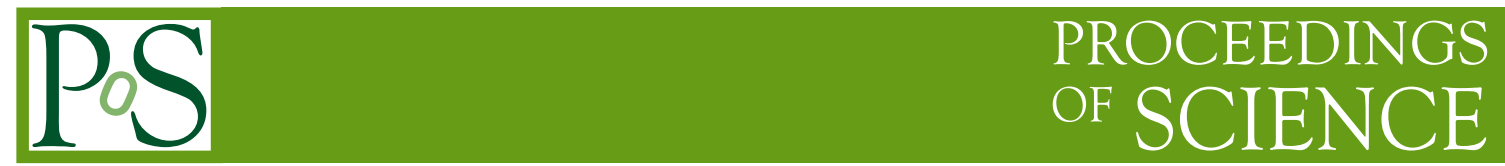

\title{
Sensitivity to EFT operators from top and Higgs measurements
}

\author{
Peter Galler ${ }^{a, *}$ \\ ${ }^{a}$ SUPA, School of Physics and Astronomy, University of Glasgow, Glasgow, G12 8QQ, UK \\ E-mail: peter.galler@glasgow.ac.uk
}

In this contribution we present a perspective on the current status of LHC measurements and their interpretation in terms of the Standard Model Effective Field Theory (SMEFT) in the top and Higgs sectors. In particular, we compare in two examples the sensitivity of effective field theory (EFT) fits performed by experimental collaborations based on individual measurements and the sensitivity of global EFT fits using multiple experimental results.

The Eighth Annual Conference on Large Hadron Collider Physics-LHCP2020

25-30 May, 2020

online

${ }^{*}$ Speaker 


\section{Introduction}

Effective field theory is an important tool for studying physics beyond the Standard Model (BSM). In particular, the SMEFT (see Ref. [1] for a recent review) can be seen as a systematic renormalisable (order by order) gauge invariant parametrisation of high scale new physics in terms of operators of mass dimension $d>4$. These higher dimensional operators are suppressed by $\Lambda^{4-d}$ where $\Lambda$ is the new physics scale. In a top-down approach we arrive at the low energy EFT by integrating out the heavy states of the underlying UV theory. Specific UV theories generate a particular set of effective operators at low energies. The Wilson coefficients of these operators can be expressed in terms of parameters of the UV theory. However, the current experimental situation at the LHC does not point to any specific UV completion of the standard model (SM). Hence, it is appropriate to follow the bottom-up approach where the SM Lagrangian is expanded in terms of $\Lambda^{4-d}$. Each term in this expansion contains all possible operators of mass dimension $d>4$ that are invariant under the SM symmetries. Their Wilson coefficients are free parameters since we assume no knowledge about the UV theory. The first order that is relevant for top and Higgs processes at the LHC is $d=6$ which is suppressed by $\Lambda^{-2}$. In principle it is possible to consider higher orders in the EFT expansion but the number [2] of operators quickly becomes overwhelming such that most phenomenological studies truncate the series after $\Lambda^{-2}$ taking only effective operators with $d \leq 6$ into account.

In the following we will compare constraints on Wilson coefficients obtained a) by experimental analyses directly and b) by global parameter fits taking multiple experimental results into account. We discuss one example for four-top production where Wilson coefficients of four-fermion operators are fitted and a second example where $H \rightarrow Z Z^{*} \rightarrow 4 \ell$ is used to fit Wilson coefficients relevant for Higgs processes.

\section{Comparison}

Four-top production is a particularly interesting process to be studied using EFT because it provides sensitivity to four-fermion operators that couple four top quarks. In addition, due to its large threshold it probes a high-energy region where EFT effects can become more prominent. In Ref. [3] the CMS collaboration used their four-top production cross section measurement to fit four Wilson coefficients given in the Warsaw basis [4]. The plot on the left-hand side of Fig. 1 shows in blue (individual) and purple (marginalised) the experimental bounds at $95 \%$ confidence level (CL). In addition, the same plot shows bounds from a global top EFT fit [5] obtained by the SMEFiT collaboration. In this fit many different top quark processes, observables and measurements were taken into account as well as additional effective top quark operators. While the individual bounds for CMS and SMEFiT are compatible there is some discrepancy in the marginalised bounds. The reason is that the global fit is sensitive to correlations between the four-fermion operators that couple only heavy quarks (bottom and top) and four-fermion operators that couple two light quarks with two top quarks. Due to these correlations the marginalised bounds obtained by SMEFiT are significantly weaker than the individual bounds. This is an illustrative example for the importance of global fits to obtain a realistic picture of the bounds on Wilson coefficients in the most general scenario where all Wilson coefficients are considered free parameters. 
Next we compare bounds on effective Higgs operators obtained either directly by the experimental analysis [6] or by a global fit performed in Ref. [7]. The results are jointly presented on the right-hand side of Fig. 1. The global fit incorporates electro-weak precision data as well as results from multiple Higgs processes measured in LHC run I and II. The experimental analysis, however, studies Higgs production in one particular decay mode, $H \rightarrow Z Z^{*} \rightarrow 4 \ell$. One can see clearly in Fig. 1 that for most of the Wilson coefficients the global fit is one to two orders of magnitude more sensitive than the results from the experimental analysis. This illustrates that including as many relevant experimental results as possible increases the constraining power of the global fit. However, the advantage of the individual experimental analysis in Ref. [6] is its usage of a signal acceptance which depends on the Wilson coefficients. This can have a significant impact on the fit and the resulting bounds. The bounds obtained by the global fit are very tight compared to the ATLAS results and therefore the acceptance is indeed SM-like. However, the question remains whether these bounds would have been different if an EFT dependent acceptance had been used in the global fit.
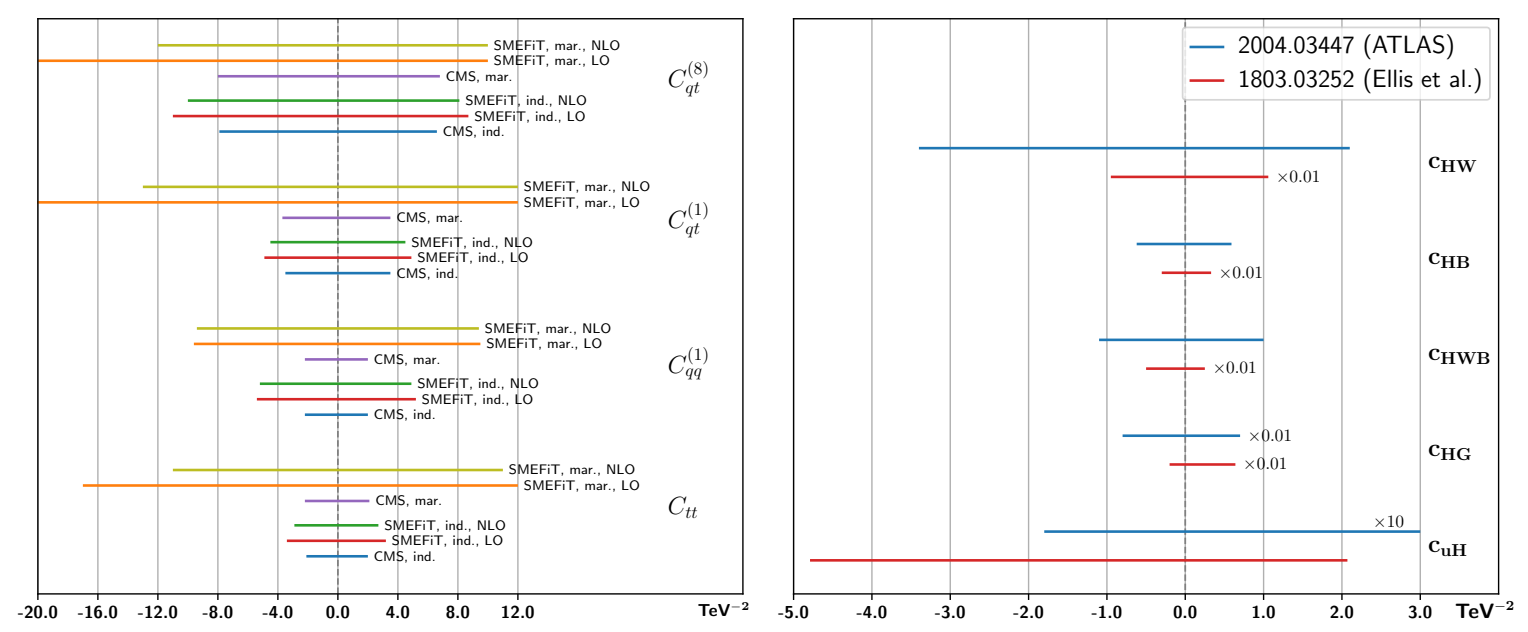

Figure 1: Left: Comparison of $95 \%$ CL bounds on four-fermion Wilson coefficients with four heavy quarks (bottom and top) between the SMEFiT collaboration [5] and CMS [3]. LO denotes results for a leading-order treatment of EFT and $N L O$ denotes results where next-to-leading order QCD corrections within the EFT were taken into account. Individual bounds ('ind.') are obtained by setting all other Wilson coefficients to zero while for marginalised bounds ('mar.') all considered operators are allowed to vary. Right: Individual bounds for Wilson coefficients which are relevant for Higgs processes. Blue lines represent 95\% CL bounds from ATLAS [6] and red lines represent bounds from a global fit in Ref. [7]. Some bounds where scaled for better visibility.

\section{Conclusion}

The top and Higgs EFT examples above illustrate that a global approach is necessary in order to obtain a reliable picture of EFT constraints in the SMEFT. First, a global approach includes more operators and therefore becomes sensitive to their correlations and second, more processes included in the global fit lead to tighter bounds on the Wilson coefficients. Recent experimental efforts for instance in Ref. [8] show a promising path to more global analyses performed by experimental 
collaborations.

Global fits have the potential to be much more than an interpretation of the available experimental data. They can be used as a testing ground for new observables and (rare) processes to determine their impact on the global EFT picture. Hence, global fits can help to direct experimental efforts to constrain the SMEFT most effectively by identifying those observables that provide the highest sensitivity to the Wilson coefficient.

More precise measurements also require a better understanding of aspects like EFT dependent signal acceptance, EFT contributions to background processes and/or parton distribution functions, etc. Therefore, only a combined approach with dedicated experimental EFT analyses taking these effects into account and global fits using state-of-the-art theoretical predictions within the SM and EFT will be able to extract the maximal information from the LHC measurements in terms of EFT constraints. As a final remark: While global EFT fits are designed for a bottom-up approach they can also be used to constrain concrete realisations of new physics. This can result in very tight constraints on the parameters of the UV model due to the correlations between the Wilson coefficients that are induced by that model (for a recent example, see Ref. [9]). Hence, the machinery and power of global EFT fits can also be applied to obtain valuable information about plausible UV completions and their parameter spaces.

\section{References}

[1] I. Brivio and M. Trott, The Standard Model as an Effective Field Theory, Phys. Rept. 793 (2019) 1-98, arXiv: 1706.08945 [hep-ph].

[2] B. Henning, X. Lu, T. Melia, and H. Murayama, 2, 84, 30, 993, 560, 15456, 11962, 261485, ...: Higher dimension operators in the SM EFT, JHEP 08 (2017) 016, arXiv: 1512.03433 [hep-ph]. [Erratum: JHEP 09, 019 (2019)].

[3] CMS Collaboration, A. M. Sirunyan et al., Search for the production of four top quarks in the single-lepton and opposite-sign dilepton final states in proton-proton collisions at $\sqrt{s}=13$ TeV, JHEP 11 (2019) 082, arXiv: 1906.02805 [hep-ex].

[4] B. Grzadkowski, M. Iskrzynski, M. Misiak, and J. Rosiek, Dimension-Six Terms in the Standard Model Lagrangian, JHEP 10 (2010) 085, arXiv: 1008 . 4884 [hep-ph].

[5] N. P. Hartland, F. Maltoni, E. R. Nocera, J. Rojo, E. Slade, E. Vryonidou, and C. Zhang, A Monte Carlo global analysis of the Standard Model Effective Field Theory: the top quark sector, JHEP 04 (2019) 100, arXiv: 1901.05965 [hep-ph].

[6] ATLAS Collaboration, G. Aad et al., Higgs boson production cross-section measurements and their EFT interpretation in the $4 \ell$ decay channel at $\sqrt{s}=13 \mathrm{TeV}$ with the ATLAS detector, arXiv:2004.03447 [hep-ex].

[7] J. Ellis, C. W. Murphy, V. Sanz, and T. You, Updated Global SMEFT Fit to Higgs, Diboson and Electroweak Data, JHEP 06 (2018) 146, arXiv : 1803.03252 [hep-ph].

[8] CMS Collaboration, Using associated top quark production to probe for new physics within the framework of effective field theory, CMS-PAS-TOP-19-001.

[9] S. Brown, C. Englert, P. Galler, and P. Stylianou, Electroweak Top Couplings, Partial Compositeness and Top Partner Searches, arXiv:2006.09112 [hep-ph]. 